\title{
13.4 High frequency of CNS involvement in linear scleroderma of the face
}

\author{
M Parolin 1 , R Russo ${ }^{2}$, F Corona ${ }^{3}$, SKF de Oliveira4, EM Nowakowska ${ }^{5}$,

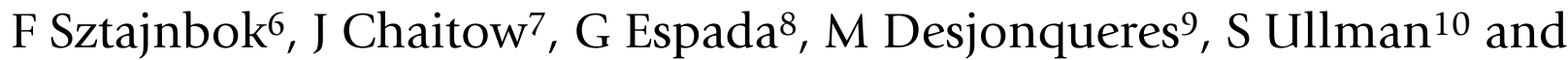 \\ F Zulian*1
}

\begin{abstract}
Address: ${ }^{1}$ University of Padua, Padua, Italy, ${ }^{2}$ Hospital de Pediatria Juan P. Garrahan, Buenos Aires, Argentina, ${ }^{3}$ Clinica Pediatrica II De Marchi, Milan, Italy, ${ }^{4}$ Instituto de Puericultura e Pediatria Martagao Gesteira, Rio de Janeiro, Brazil, ${ }^{5}$ Institute of Rheumatology, Warsaw, Poland, ${ }^{6} \mathrm{Hospital}$ Universitario Pedro Ernesto, Rio de Janeiro, Brazil, ${ }^{7}$ The Children's Hospital Westmead, Sydney, Australia, ${ }^{8}$ Hospital de Ninos Ricardo Gutierrez, Buenos Aires, Argentina, ${ }^{9}$ Hopital E Herriot, Lyon, France and ${ }^{10}$ Bispebjerg Hospital, Copenhagen, Denmark

* Corresponding author
\end{abstract}

from 15th Paediatric Rheumatology European Society (PreS) Congress

London, UK. 14-17 September 2008

Published: 15 September 2008

Pediatric Rheumatology 2008, 6(Suppl I):S27 doi:10.II86/I546-0096-6-SI-S27

This abstract is available from: http://www.ped-rheum.com/content/6/SI/S27

(C) 2008 Parolin et al; licensee BioMed Central Ltd.

\section{Background}

Single case reports have reported neurological symptoms or cerebral imaging abnormalities in linear scleroderma of the face (LSF). Aim of the study was to investigate the frequency and clinical features of CNS involvement in a large cohort of patients with LSF.

\section{Methods}

Patients included in the JUvenile Scleroderma International (JUSI) database of PRES with LSF were evaluated. Clinical and laboratory information were analyzed in order to define frequency, type, timing of onset and possible risk factors of CNS involvement.

\section{Results}

121 patients with LSF, followed at 12 centers entered the study. F:M ratio was 2, 1:1, mean age at onset 5, 8 years.

Among 77 patients whose we obtained complete clinical and instrumental updated documentation (MRI, CT scan and EEG), 46 (59.7\%) had CNS involvement. 33 (42.9\%) presented with symptoms such as epilepsy in $16(20.8 \%)$, chronic headache in $11(14.3 \%)$, hemiparesis with or w/o epilepsy in $3(3.9 \%)$ or behavioural disorders in $3(3.9 \%)$. Five patients $(15.1 \%)$ had seizures before the onset of the skin changes, $39.4 \%$ presented neurologic symptoms by the first year and $84,8 \%$ by 5 years since the disease onset. 13 asymptomatic patients presented only EEG or cerebral imaging abnormalities. All these findings were ipsilateral to the skin lesion. No significant correlation was found between CNS involvement and autoantibodies, age at onset, gender, family history for autoimmune diseases and environmental factors.

\section{Conclusion}

LSF is frequently associated with CNS abnormalities. A careful clinical and instrumental evaluation should be recommended for every patient at onset and during the follow-up. 